\title{
From the thermal and kinematical full-field measurements to the analysis of deformation mechanisms of NiTi SMAs
}

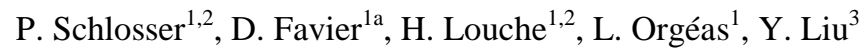 \\ ${ }^{1}$ Université de Grenoble/CNRS, 3S-R, BP 53, 38041 Grenoble Cedex 09, France \\ ${ }^{2}$ Université de Savoie, SYMME, BP 80439, 74944 Annecy le Vieux cedex, France \\ ${ }^{3}$ The University of Western Australia. School of Mechanical Engineering, Crawley, WA 6009, Australia
}

\begin{abstract}
Intensive experimental investigations have been carried out to understand deformation mechanisms associated with the superelasticity of polycrystalline nickel-titanium shape memory alloys. This paper analyses results of superelastic tensile tests on thin plates and thin walled tubes of NiTi. During the deformation, thermal and kinematical full-field measurements were simultaneously carried out using a visible and an infrared camera. Heat source fields were estimated from the thermal measurement during both homogeneous and localized stages of deformation of these tests. Local heat energy values were then obtained by integrating heat sources over time for any given location on the sample. This provides in-situ local calorimetric measurements similar to a virtual local differential scanning calorimeter all along the specimens. Deformation mechanisms can be analyzed from the simultaneous knowledge of heat source-strain rate fields or of heat energy-strain fields. It is shown that forward and reverse transformation deformation mechanisms are involved in all homogeneous stages usually considered as elastic deformation of austenitic and of martensitic phases. The kinematic and morphology of deformation localization during deformation stages over the stress plateaus are also investigated. It is shown that forward (loading) and reverse (unloading) transformations are not complete inside the localized bands.
\end{abstract}

\section{Introduction}

Kinematical and thermal full field measurements are useful experimental techniques to study the complex behavior of shape memory alloys (SMA). Thermal $([1,2]$ for example), kinematical ([3] for example) and simultaneous kinematical and thermal [4] fields have been obtained during superelastic tensile tests on nickeltitanium samples. Most of the previous studies focused mainly on localization effects occurring during forward and reverse deformation stages over the stress plateaus, observed by monitoring the temperature and strain fields.

This paper presents a study of thermal and kinematical field measurement to analyze more in depth superelastic tensile tests of NiTi SMAs, both during uniform and localized deformation stages. The paper (i) presents simultaneous kinematics and thermal full field measurements obtained from a visible and an infrared camera, and (ii) presents calorimetric and mechanical analyses to elucidate the deformation mechanisms. Images are recorded during tensile tests on superelastic NiTi plate and tube samples. A processing method was applied to estimate, from the thermal fields, the heat sources associated with the material deformation (thermoelasticity, intrinsic dissipation or phase transformation). Local heat energy values are then obtained by integrating heat sources over time for any given location on the sample. This provides in-situ local calorimetric measurements similar to a local DSC all along the specimens.

\section{Experimental set-up}

\subsection{Materials and testing}

Tensile tests on Ti-50.6 at\% Ni plates (width $7.14 \mathrm{~mm}$, thickness $0.5 \mathrm{~mm}$ ) and on Ti-50.8 at\% Ni tubes (outside diameter $10 \mathrm{~mm}$, wall thickness $0.5 \mathrm{~mm}$ ) were performed at room temperature $T_{0}$. DSC measurements of the two alloys are plotted in Fig. 1. The plate alloy exhibited a simple Austenite (A) $\rightarrow$ Martensite (M) transformation

\footnotetext{
a e-mail: denis.favier@grenoble-inp.fr
}

This is an Open Access article distributed under the terms of the Creative Commons Attribution-Noncommercial License (http://creativecommons.org/licenses/by-nc/3.0/), which permits unrestricted use, distribution, and reproduction in any noncommercial medium, provided the original work is properly cited. 
during cooling and $\mathrm{M} \rightarrow \mathrm{A}$ during heating. The tube sample exhibited an intermediate $\mathrm{R}$ phase both during cooling and heating. For both alloys, the austenite finish temperature is below the room temperature.

\subsection{Temperature and strain field measurements}

During the tests, temperature and displacement fields were simultaneously measured on the sample surface using an infrared and a visible camera. The sample surface was coated with high emissivity black paint with a white texture on top. Such coating is necessary for the use of a digital image correlation (DIC) data processing method to compute the displacement fields and is compatible with infrared thermography measurements. More information on this experimental set-up can be found in references [4-6].

a) Plate

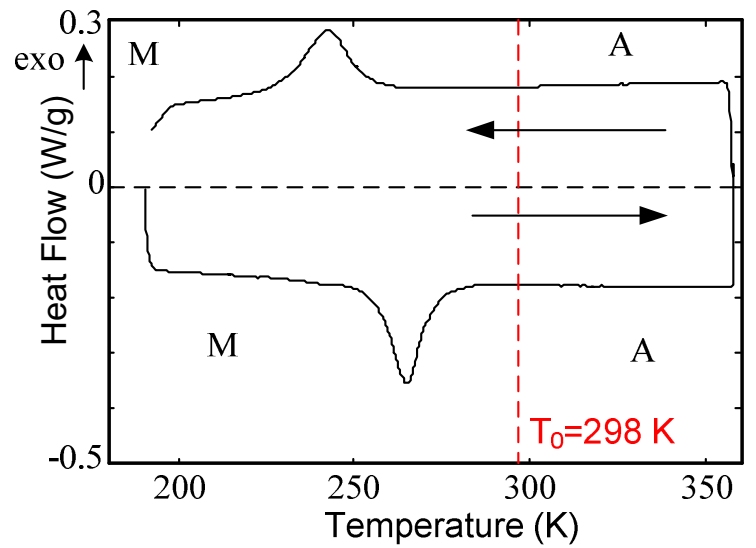

b) Tube

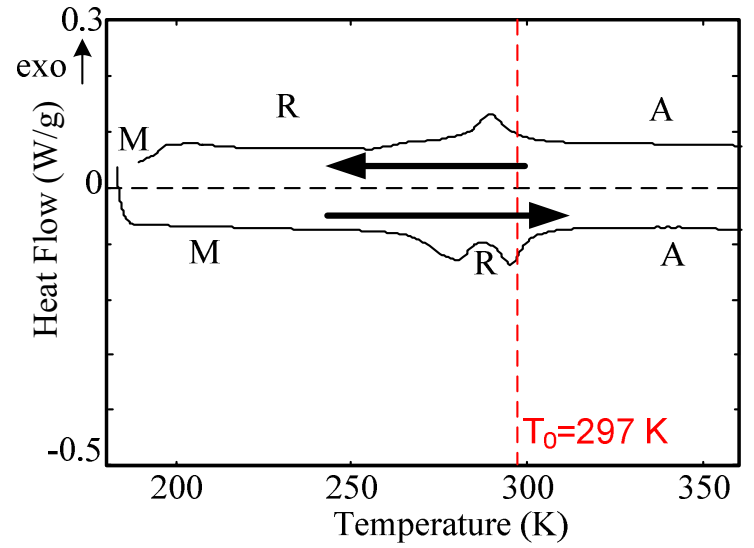

Fig. 1. DSC measurements of a) the Ti- 50.6 at $\% \mathrm{Ni}$ plate and b) the Ti- 50.8 at $\% \mathrm{Ni}$ tube. The vertical dashed line shows the testing (room) temperature $\mathrm{T}_{0}$.

The displacement field $\vec{u}$ was obtained for every material grid cell on the sample surface using a digital visible camera (Hamamatsu, 12 bit, 1280x1024 pixels) and a DIC processing software (7D software, [7]). The Green-Lagrange strain fields were estimated from the displacement field $\vec{u}$ either in Lagrangian (as function of the initial position $M_{0}$ of the material particle) or in Eulerian (as function of the current position $\mathrm{M}$ of the material particle) description. A mean nominal axial strain $\varepsilon=\Delta l / l_{0}$ was measured from the displacement fields using the relative displacement $\Delta l$ of two particles $\mathrm{A}$ and B located at the extremities of the gauge length. $l_{0}=58$ $\mathrm{mm}$ ( $l_{0}$ is the initial distance between these two points). A fast multidetector infrared camera (CEDIP Jade III MW, 14 bit, 320x240 pixels, thermal resolution $20 \mathrm{mK}$ ) was used to measure the temperature field $T(M, t)$ in each spatial point $M$.

A first temporal synchronization was realized in order to synchronize the three time bases associated with the mechanical measurements (load) and the thermal and kinematical fields' data. Then, a second spatial synchronization of the thermal and kinematic fields was proposed to obtain the thermal and kinematical values of the same material particles (of initial and current positions $M_{0}$ and $M$, respectively). Such synchronization is necessary during a mechanical test in order to estimate properly the temperature temporal variation for a given material particle and thereafter the heat energy by time integration of the heat sources for given material particles. More details on this method are given in [6] and will be presented in [8].

\subsection{Heat sources estimation}

Due to thermal conduction, temperature fields are often difficult to analyze. One needs to estimate the heat sources in order to (i) better analyze the nature of the deformation mechanisms, (ii) observe the position and kinetics of the phase transformation and (iii) to perform a local DSC when estimating the specific heat energy by time integration of the heat sources. The method to obtain estimations of the heat source field from the thermal field was explained in [9] for plate geometry and [5] for tube geometry. For thin plates or thin walled tubes it is assumed that the temperature and the associated heat sources are uniform in the thickness direction. Then, the heat sources $s_{i}$ (volumic heat power in $\mathrm{W} / \mathrm{m}^{3}$ ) can be obtained through an estimation of the left hand side of the 2D heat diffusion equation:

$$
\rho \mathrm{C}\left(\frac{\partial \theta}{\partial \mathrm{t}}+\frac{\theta}{\tau_{t h}}\right)-k \Delta_{2} \theta=\mathrm{s}_{\mathrm{i}}
$$


where $\theta=T-T_{0}$ is the temperature variation, $\rho$ the mass density, $C$ the specific heat, $k$ the heat conduction and $\tau_{\mathrm{th}}$ a time constant (in s) modeling the back and front face heat losses by convection and radiation.

When the heat sources are uniform and $\theta<<T_{0}$, some simplifications of equation (1) can be made [9], so that the heat conduction equation reads:

$$
\rho \mathrm{C}\left(\frac{\partial \theta}{\partial \mathrm{t}}+\frac{\theta}{\tau_{e q}}\right)=\mathrm{s}_{\mathrm{i}}
$$

$\tau_{e q}$ represents a characteristic time reflecting the overall heat losses. This simplified model was used in [4] to study superelastic NiTi tubes during homogeneous deformation stages. It is well known that material parameters such as $k$ and $C$ are strongly dependent on the phase type [10,11]. In this paper, a mean value was considered for the specific heat $\left(\mathrm{C}=590 \mathrm{Jkg}^{-1} \mathrm{~K}^{-1}\right)$ and the two following values were taken for the thermal conductivity of phases A and M: $k=k_{A}=18 \mathrm{Wm}^{-1} \mathrm{~K}^{-1}$ and $k=k_{M}=8 \mathrm{Wm}^{-1} \mathrm{~K}^{-1}$.

In this paper, the first 2D model (eq. (1)) has been used to compute heat sources. The first study concerns the uniform deformation preceding the stress plateau; thus, constant values for the material parameters $k$ and $C$ in equation (1) have been used, equal to $k_{A}$. The second study deals with deformation localization phenomena on tubes; thus, heat sources were estimated using a model taking into account the important difference of thermal conductivity $k$ between the $\mathrm{A}\left(k=k_{A}\right)$ and $\mathrm{M}$ phases $\left(k=k_{M}\right)$.

\section{Example of Results}

\subsection{Uniform deformation preceding the superelastic tensile stress plateau of a NiTi plate}

Deformation localization phenomena during superelastic tensile test is preceded by a uniform deformation stage, often considered as purely elastic. This first apparent "elastic" loading was studied on NiTi tubes in [4]. Here, we propose to analyze this same homogeneous stage but in a plate NiTi sample exhibiting no R phase. The stressstrain curves of two tensile tests (noted $\mathrm{P}_{1}$ and $\mathrm{P}_{2}$ ) conducted on the plate are presented in Fig. 2a. Figure $2 \mathrm{~b}$ shows the details of test $\mathrm{P}_{1}$ and indicates the positions of characteristic times $a$ to $d$. Deformation localization started at time $b$ (as evidenced by the stress plateau in test $\mathrm{P}_{2}$ ), and no localization was observed within the gauge length of test $P_{1}$ either on the temperature or strain maps. Due to the rectangular shape of the sample, the localization had started within the grips between times $b$ and $c$, outside the observation area.
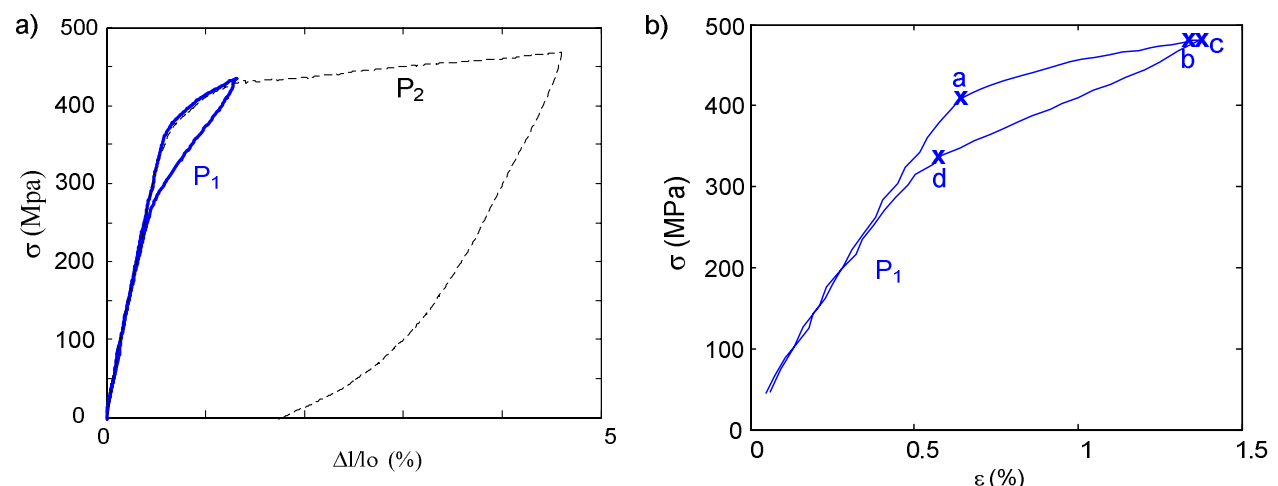

Fig. 2. a) Nominal stress-strain curve of tensile tests performed on plates $P_{1}$ and $P_{2}$. b) Close-up showing the response of $P_{1}$

Specific heat sources $\dot{q}=\frac{s_{i}}{\rho}$, estimated from eq. (2), were found to be uniform during loading and unloading. As previously used in [4], heat source $\dot{q}$ includes $\dot{q}_{\text {thel }}$, which is the usual thermoelastic coupling which can be estimated from $\dot{q}_{\text {thel }}=\frac{-\alpha T_{0} \dot{\sigma}}{\rho}$, where $\alpha=1110^{-6} \mathrm{~K}^{-1}$ is the thermal expansion coefficient of the austenite, $\rho=6400 \mathrm{~kg} / \mathrm{m}^{3}$ the mass density and $\dot{\sigma}$ the axial stress rate.

Fig. 3a shows the difference $\dot{q}-\dot{q}_{\text {thel }}$ versus the axial stress for test $P_{l}$. During loading, up to time $a$ (about $350 \mathrm{MPa}$ ) the heat sources remain weak but positive (exothermic heat sources). After time $a$ and up to the end of loading the heat sources increase quickly up to $1.2 \mathrm{~W} / \mathrm{g}$. During unloading, endothermic heat sources are measured, high from $\mathrm{c}$ to $\mathrm{d}$, and weaker (but not null) from $\mathrm{d}$ to the end of unloading. These exothermic heat sources during loading (endothermic during unloading) confirm the presence of other deformation mechanisms than pure elasticity within this strain range. The heat energy, excluding the thermoelastic energy and computed with a temporal integration of the heat sources in each material particle, is plotted in Fig. 3b. Heat energy 
increases with stress at above $400 \mathrm{MPa}$ and decreases during unloading. The final small but positive value at the end of unloading can be interpreted as remaining martensite and/or intrinsic dissipation.
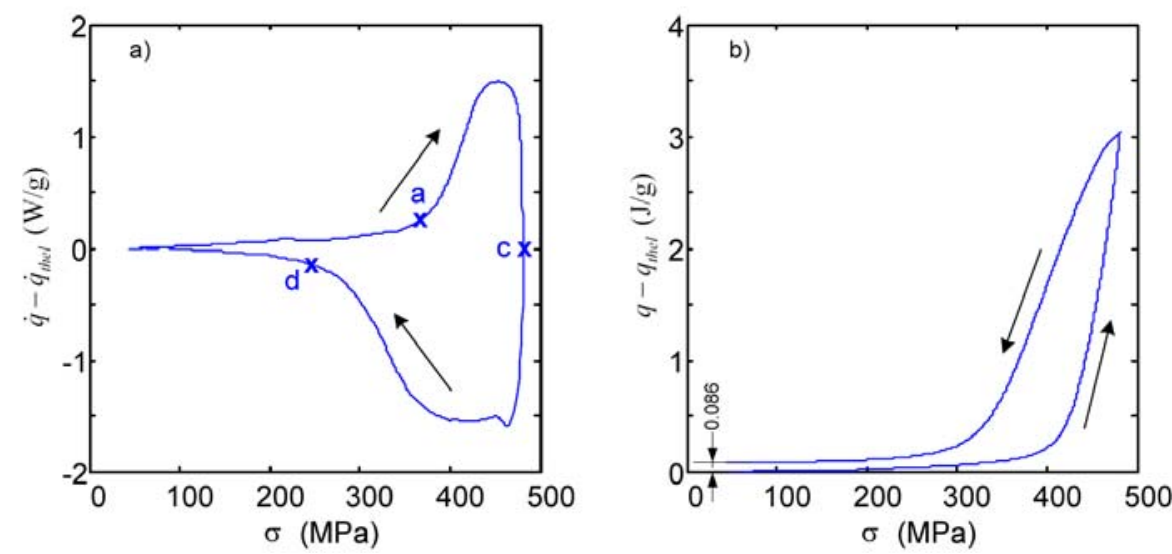

Fig. 3: (a) Heat source difference $\dot{q}-\dot{q}_{\text {thel }}$ vs. axial stress for test $P_{l}$. (b) Heat energy difference obtained from a temporal integration of the heat sources

As proposed in [4] the following ratio was computed in order to interpret the heat source intensities:

$$
\frac{\dot{q}_{t r}}{\dot{\varepsilon}_{t r}}=\frac{\dot{q}-\dot{q}_{t h e l}}{\dot{\varepsilon}-\dot{\varepsilon}_{e}}=\frac{\dot{q}+\frac{\alpha T_{0} \dot{\sigma}}{\rho}}{\dot{\varepsilon}-\frac{\dot{\sigma}}{E}},
$$

where $\dot{\varepsilon}_{t r}$ and $\dot{\varepsilon}_{e}$ are the transformation and elastic strain rates, and $E$ is the Young's modulus of the A phase. This last parameter was chosen equal to $110 \mathrm{GPa}$, in the range given from microscopic observations in $[12,13]$, respectively for $\mathrm{A}$ and $\mathrm{M}$ phase.

Noting $\dot{f}$ the martensite fraction rate and assuming the following proportionality relations $\dot{q}_{t r}=\dot{f} \Delta H_{t r}$ and $\dot{\varepsilon}_{t r}=\dot{f} \Delta \varepsilon_{t r}$, where $\Delta H_{t r}$ and $\Delta \varepsilon_{t r}$ are the latent heat and the strain for the complete transformation respectively, an other ratio can be computed in order to estimate $\Delta H_{t r}$ :

$$
\frac{\Delta H_{t r}}{\Delta \varepsilon_{t r}}=\frac{\dot{q}_{t r}}{\dot{\varepsilon}_{t r}}
$$

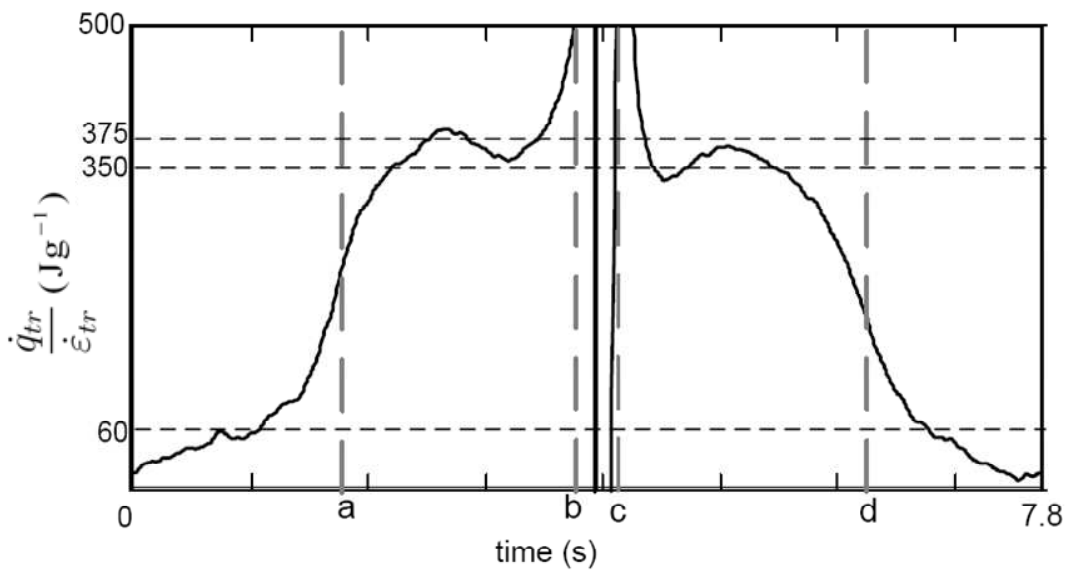

Fig. 4. Evolution of the $\frac{\dot{q}_{t r}}{\dot{\varepsilon}_{t r}}$ ratio

This ratio, plotted in Fig. 4, shows two "plateaus" during the loading and unloading stages, confirming the presence of two deformation mechanism stages up to time $b$. The first plateau at around $60 \mathrm{~J} / \mathrm{g}$ cannot be associated with elasticity or phase transformation mechanism, and it was interpreted in [6] as being associated with precursor phenomena [14]. The plateaus at between $375 \mathrm{~J} / \mathrm{g}$ and $350 \mathrm{~J} / \mathrm{g}$ were associated with the martensic transformation A $\leftrightarrow \mathrm{M}$. With $\Delta \varepsilon_{t r}=8 \%, \Delta H_{t r}$ was then estimated to be approximately $30 \mathrm{~J} / \mathrm{g}$. This value of the latent heat of stress induced martensitic transformation is higher than that of the thermally induced 
transformation obtained by DSC (16 J/g in Figure 1). This result has already been observed in [15] during the reverse $\mathrm{M} \rightarrow \mathrm{A}$ transformation and in [4] for the forward transformation.

\subsection{Study of the localized stage in NiTi tubes}

Fig. 5b-f show the full field views of the tube surface, including the axial component of the Green-Lagrange strain field $E_{x x}(P, t)$, the temperature variation field $\theta(P, t)$ and the heat source field $s_{M}(P, t)$ obtained on the tube sample at characteristic times (a, b, c, d, e) given in Fig. 5a. All the data were synchronized, in time and space, and plotted on the reference configuration (i.e. as function of the initial positions $M_{0}$ of the material particles). The heat source $s_{M}$ has been estimated from eq. (1); the notation ' $M$ ' refers to the value $k=k_{M}$ chosen for the thermal conductivity.

Several comments on these first qualitative results can be made:

- $\quad$ stage 1, time a: As observed on the plate sample, the heat sources were uniform and exothermic in the whole sample.

- $\quad$ stage 2, time b and c (initiation and propagation of the strain localization): time b was taken after the beginning of the stress plateau. Localization initiated as a helical shaped band, in the upper grid at the yield point. At time b, one helical band can be observed in A and B both in the strain and heat source fields. The heat source estimation, taking into account heat conduction, increases the contrast and allows better observing the localized phase transformation. Namely, near the top grip, two heat source fronts are observed, showing the band widening (see Fig. 6c). At time c, the temperature variation was about $14 \mathrm{~K}$ but the band width was diffused, therefore difficult to analyze. Like time $\mathrm{b}$, the heat sources allow a better understanding of the localization of the current phase transformation, showing two widening fronts in the upper arrow area. As noted in the strain field some zones (see the arrows) of the tube were not transformed at the end of the stress plateau.

a)

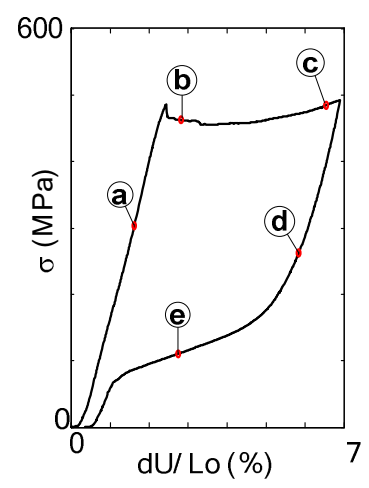

d) time c

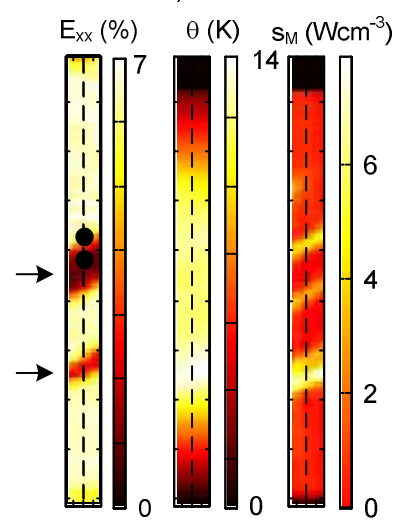

b) time a

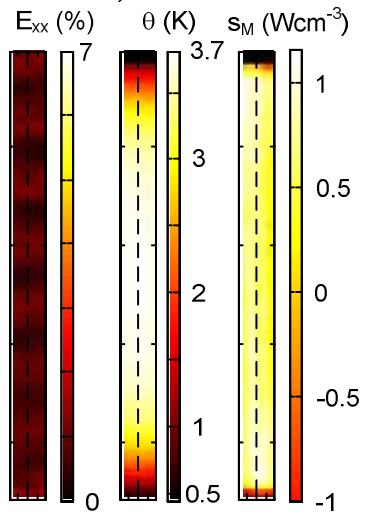

e) time $d$

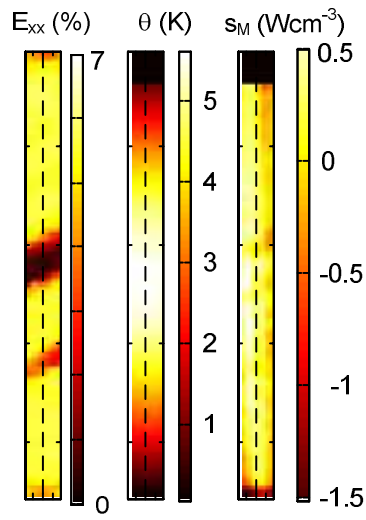

c) time $b$

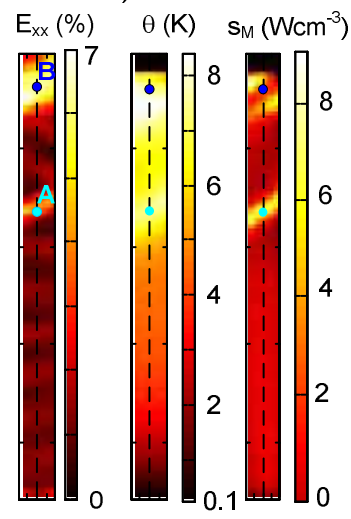

f) time e

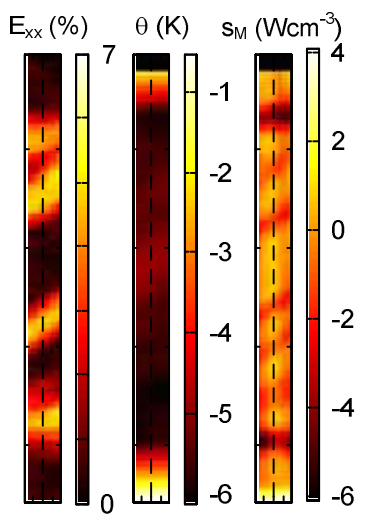

Fig. 5. Tensile test at $\dot{U} / L_{0}=2.310^{-4} \mathrm{~s}^{-1}$.

a) Nominal stress-strain response with the position of five selected times.

b) Green Lagrange axial strain field $E_{x x}(P, t)$, temperature variation field $\theta(P, t)$ and heat sources field $s_{M}(P, t)$ at time $a$

c) to f) same fields at times $b$ to $e$. 
- $\quad$ stage 3, time d: at the beginning of unloading, the strain field was heterogeneous but decreased homogeneously. Conversely, heat sources were homogeneous and negative, revealing homogeneous reverse transformation.

- $\quad$ stage 4, time e: during the unloading stress plateau, the strain field was once again localized, associated with reverse localized phase transformation. The position of the strain gradients corresponded to endothermic localized heat sources, whereas the temperature field was below room temperature and again difficult to analyze.

For a better analysis of these fields, axial component of the Green-Lagrange strain $E_{x x}(X, Y=0, t)$ and heat source $s_{M}(X, Y=0, t)$ along one tube generating line $(\mathrm{Y}=0)$ are plotted in Fig. $6 \mathrm{~b}$ and $\mathrm{c}$ at selected times marked in Fig. 6a. During the stress plateau (stage 2, times $b$ and $c$ ), the strain fields are strongly localized. The heat source profiles show clearly peak values at the position of high strain gradients and correspond to the position of the more active phase transformations. Moreover, the heat source peaks have higher amplitude when the band is propagating rather than widening.

Specific heat energy $q$, obtained by a temporal integration of the heat sources in each particle $P$, is plotted in Fig. 6d. The energy profiles are almost identical to those of the strain. The energy profiles reach at the end of the stress plateau a maximum of $\sim 36 \mathrm{~J} / \mathrm{g}$ within the deformed area. Once again, this value is higher than the heat determined from the DSC measurement [15]. Quantification of transformation latent heat by this technique will require further validation via careful calibration against well known systems. Such calibration is being conducted currently.
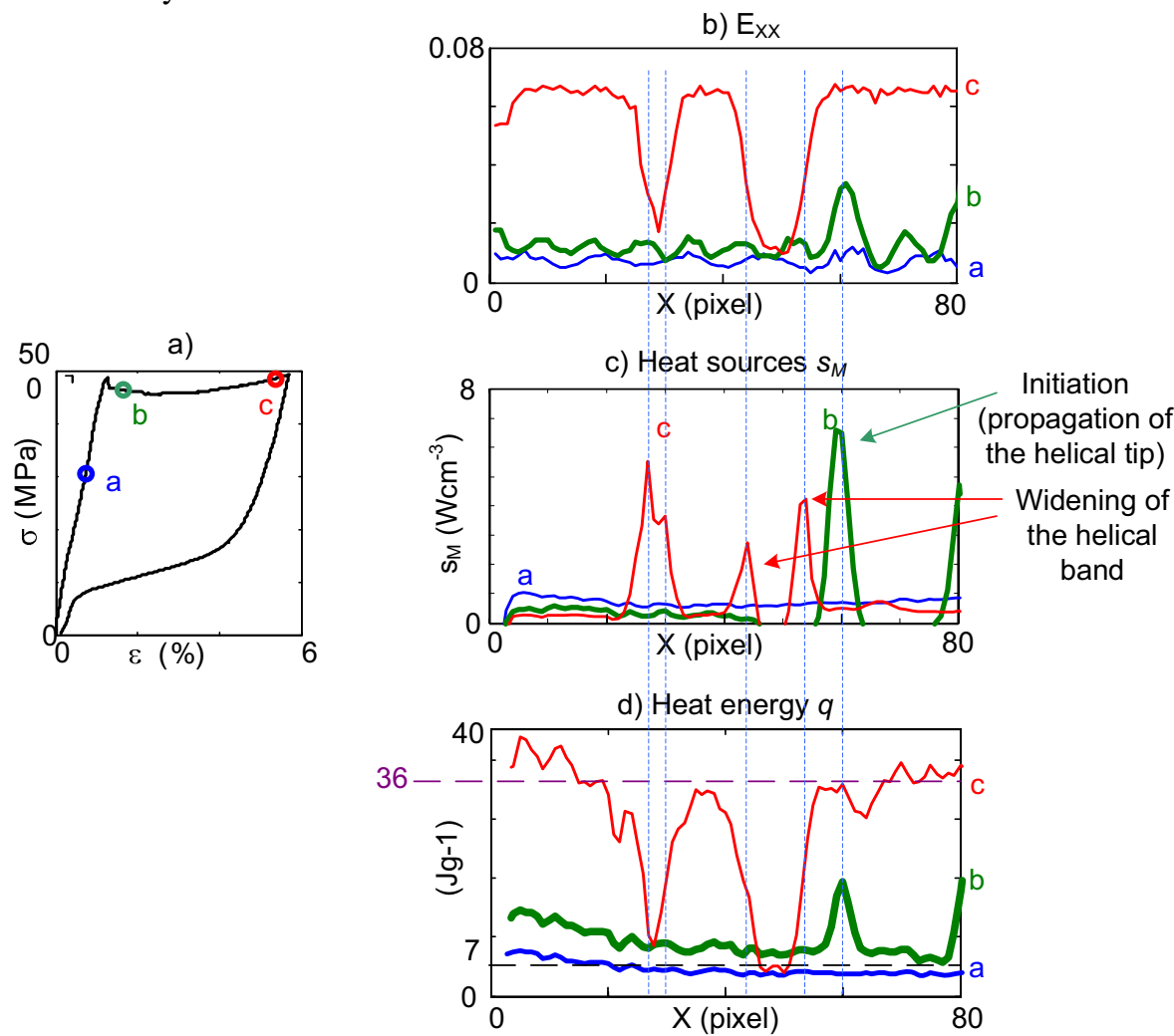

Fig. 6. a) Stress-strain curve with the position of three selected times during loading. b) $E_{x x}$ strain axial profiles at the three selected times. c) Heat sources axial profiles estimated at the same times. d) Energy estimated at the same time.

Spatiotemporal evolutions of $E_{x x}$ and $q$ along the same axial profile are plotted in Fig. 7. Since at the end of the loading the transformation was not completed, a reduced zone in the middle of the sample was still austenitic at the end of the plateau (time $c$ ). The energy in this area is around $7 \mathrm{~J} / \mathrm{g}$. The resemblance between these physical data, obtained with two independent raw data (visible images and infrared images), is noticeable. The heat energy, measured locally during the test, can be considered as "local in-situ DSC" of the material.

\section{Conclusions}

An original experimental set-up, with spatio-temporal synchronized kinematic and thermal field measurements has been used to study the thermomechanical behaviour of superelastic NiTi plate and tube samples. Heat sources, estimated from the thermal fields and a 2D heat diffusion model, were also presented. They give additional information on the material behaviour. Two examples were presented. The first one, on a NiTi plate 
without $\mathrm{R}$ phase, shows an analysis of the first homogeneous loading, before the stress plateau. Heat sources estimated during this stage indicated that the material behaviour was not pure elasticity but a mix of elasticity, transformation and some precursor phenomena. In the second example on a NiTi tube sample, the localized phase transformation was studied by means of measuring the axial strain, temperature variation and heat source fields. The localization proceeded along two modes: first a helical band initiated in a grip then turned around the tube; second, a widening of the helical front appeared clearly on the heat source fields. Finally, heat energy distribution in the tube and over the time was presented and looked as axial strain field distribution. Such energy result can be considered as "local in-situ DSC". This technique provides us, for the first time, a real opportunity to determine the latent heat of stress-induced martensitic transformation, which is considered different from that of thermally induced martensitic transformation as conventionally determined by DSC. The availability of such data is critically important for superelasticity related theoretical calculations and engineering designs.

a)

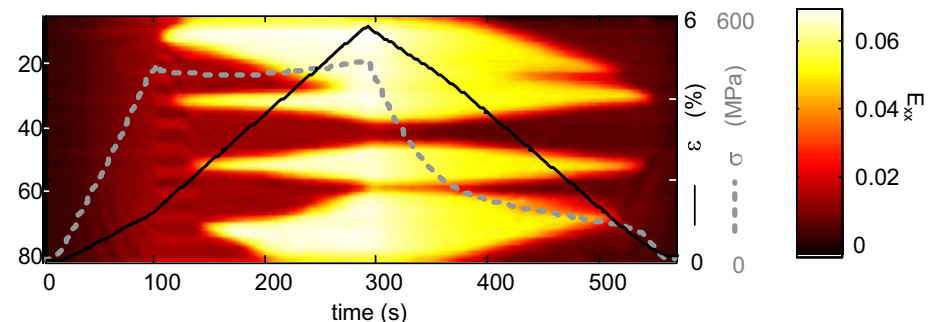

b)

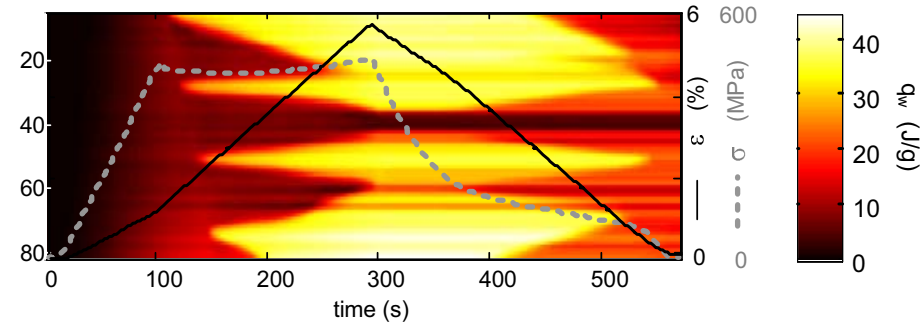

Fig. 7. Spatiotemporal representation along the tube axis of: a) the Green Lagrange axial strain $E_{x x}$, and b) of the heat energy $q$. The two curves super imposed in these figures are the nominal stress $\sigma$ and the local axial strain $\varepsilon$.

\section{Acknowledgements}

We would like to thank French and Australian ministries for their support through the Program France-Australia Partenariat Hubert Curien 2008-2010 "'Lüders-like deformation Behaviour of near-equiatomic NiTi Shape Memory Alloys".

\section{References}

[1] J. Shaw and S. Kyriakides, "Thermomechanical aspects of TiNi," J. Mech. Phys. Solids, vol. 43, pp. 1243-1281, 1995.

[2] E. Pieczyska, W. Gadaj, S.P. Nowacki, and H. Tobushi, "Investigation of nucleation and propagation of phase transitions in tini sma," QIRT Journal, vol. 1, pp. 117-128, 2004.

[3] S. Daly, G. Ravichandran, and K. Bhattacharya, "Stress-induced martensitic phase transformation in thin sheets of nitinol," Acta Materialia, vol. 55, pp. 3593-3600, June 2007.

[4] D. Favier, H. Louche, P. Schlosser, L. Orgéas, P. Vacher, and L. Debove, "Homogeneous and heterogeneous deformation mechanisms in an austenitic polycrystalline ti-50.8 at.\% ni thin tube under tension. investigation via temperature and strain fields measurements," Acta Materialia, vol. 55, pp. 5310-5322, Sept. 2007.

[5] P. Schlosser, H. Louche, D. Favier, and L. Orgéas, "Image processing to estimate the heat sources related to phase transformations during tensile tests of TiNi tubes," Strain, vol. 43, pp. 260-271, Aug. 2007.

[6] P. Schlosser, Infuence of thermal and mechanical aspects on deformation behaviour of NiTi alloys. $\mathrm{PhD}$ thesis, Université Joseph Fourier, Grenoble, France, 2008.

[7] P. Vacher, S. Dumoulin, and F. Morestin, "Bidimensional deformation measurement using digital images," Proc. Instn Mech. Engrs, vol. 213, C, pp. 811-817, 1999.

[8] P. Schlosser, H. Louche, D. Favier, and L. Orgeas, "Estimation of strain rate and heat source fields during localised superelastic tensile tests on TiNi sheet samples," in Int. Conf. ESMC Euromech, Lisbonne, Portugal, September 7-11, 2009. 
[9] A. Chrysochoos and H. Louche, "An infrared image processing to analyse the calorific effects accompanying strain localisation," Int J Eng Sci, vol. 38, pp. 1759-1788, Nov. 2000.

[10] M. Faulkner, J. Amalraj, and A. Bhattacharyya, "Experimental determination of thermal and electrical properties of Ni-Ti shape memory wires," Smart Mater Struct, vol. 9, pp. 622-631, 2000.

[11] W. Huang, "On the selection of shape memory alloys for actuators," Materials and Design, vol. 23, pp. 11-19, 2002.

[12] S. Rajagopalan, A. L. Little, M. A. M. Bourke, and R. Vaidyanathan, "Elastic modulus of shape-memory NiTi from in situ neutron diffraction during macroscopic loading, instrumented indentation, and extensometry," Applied Physics Letters, vol. 86, no. 8, p. 081901, 2005.

[13] Z. Yang, W. Tirry, D. Lamoen, S. Kulkova, and D. Schryvers, "Electron energy-loss spectroscopy and first-principles calculation studies on a ni-ti shape memory alloy," Acta Materialia, vol. 56, pp. 395-404, Feb. 2008.

[14] K. Otsuka and X. Ren, "Physical metallurgy of Ti-Ni-based shape memory alloys," Progress in materials science, vol. 50, no. 5, pp. 511-678, 2005.

[15] Y. Liu and D. Favier, "Stabilisation of martensite due to shear deformation via variant reorientation in polycrystalline TiNi,” Acta Materialia, vol. 48, pp. 3489-3499, 2000. 